\title{
Calculation of dissociation temperature of nucleon using Gaussian expansion method
}

\author{
Peng Cheng, ${ }^{1}$ Qi Meng, ${ }^{1}$ Yonghui Xia, ${ }^{1}$ Jialun Ping, ${ }^{2, *}$ and Hongshi Zong ${ }^{1,3,4, \dagger}$ \\ ${ }^{1}$ Department of Physics, Nanjing University, Nanjing 210093, China \\ ${ }^{2}$ Department of Physics, Nanjing Normal University, Nanjing 210097, China \\ ${ }^{3}$ Joint Center for Particle, Nuclear Physics and Cosmology, Nanjing 210093, China \\ ${ }^{4}$ Nanjing Proton Source Research and Design Center, Nanjing 210093, China
}

(Received 4 February 2018; published 27 December 2018)

\begin{abstract}
The study of the nucleon dissociation in a hot quantum chromodynamics (QCD) medium in a constituent quark model with the help of Gaussian expansion method (GEM) is presented. This is the first time this method is applied to the dissociation problem of nucleon. The temperature-dependent potentials of a threequark system, taking as the internal energy of the corresponding system, are obtained from the free energy of the system based on Debye-Hückel theory. The lattice QCD results of free energy for heavy three-quark system are employed and extended to the light three-quark system. The Schrödinger equation for nucleon is solved with the help of GEM and the dissociation temperature of nucleon is determined according to the temperature dependence of binding energy and radius. The dissociation temperature of nucleon we calculate is about $1.16 T_{c}$ ( $T_{c}$ is the deconfinement temperature).
\end{abstract}

DOI: $10.1103 /$ PhysRevD.98.116010

\section{INTRODUCTION}

It is generally believed that a quark-gluon plasma (QGP) may be produced during relativistic heavy-ion collisions [1]. Some work shows that some bound states consisting of quarks and/or antiquarks may survive in QGP. In statistical QCD, the thermal properties of QGP can be investigated by studying the behavior of these bound states in a hot medium. In 1986, Satz pointed out that the suppression of $J / \psi$ can be recognized as a signature of QGP formation in relativistic heavy ion collisions [2]. In the past thirty years, there have been numerous theoretical and experimental researches focused on charmonium, and the investigation has been extended to many other bound states, such as bottomonium and baryons.

In high temperature and density, the interaction between quarks is screened [3] and the binding energy will decrease. As a result, the quark bound states will start to dissociate when the binding energy becomes low enough (and its radius becomes large enough). In many researches [4-7], the dissociation of quarkonium has been studied in the Schrödinger equation formalism. In this work, we will

\footnotetext{
jlping@njnu.edu.cn zonghs@nju.edu.cn
}

Published by the American Physical Society under the terms of the Creative Commons Attribution 4.0 International license. Further distribution of this work must maintain attribution to the author(s) and the published article's title, journal citation, and DOI. Funded by SCOAP. study the dissociation of nucleon in a hot QCD medium with the help of Gaussian expansion method (GEM), an efficient and powerful method in few-body system [8]. For this purpose, a constituent quark model is employed and extended to finite temperature by extending the interquark potential at zero temperature to that at finite temperatures.

The method has been applied to calculate the dissociation temperature of heavy quarkonium states [9]. The results are consistent with the ones from other work [5]. This verifies the validity and reliability of the approach. For heavy quarkonium, the temperature dependent potential can be extracted from the free energy of a heavy quarkantiquark system $F_{q \bar{q}}(r, T)$ which can be calculated in lattice QCD [10,11]. The analytical form of $F_{q \bar{q}}(r, T)$ is constructed based on Debye-Hückel theory [12], and its temperature-dependent parameters are determined by fitting the lattice data. Extending the work to the dissociation of nucleon, we need the interquark potential of nucleon in the hot medium. Based on the free energy of a heavy quark-antiquark system $F_{q \bar{q}}(r, T)$ and the relation between $F_{q \bar{q}}(r, T)$ and $F_{q q q}(r, T)[3,13,14]$, we obtain the free energy of heavy three-quark system. It should be pointed out here that the strong interaction is only related to the degree of freedom of color and has nothing to do with the degree of freedom of flavor. So the form of the strong interaction between the heavy flavor quarks and the one between the light flavor quarks is the same. Then we obtain the interquark potential of a nucleon in hot QCD medium from the free energy. After constructing the interquark potential at finite temperature, we obtain the temperature 
dependence of binding energy and radius by solving the corresponding Schrödinger equation with the help of GEM. The dissociation temperature is the point where the binding energy decreases to zero. In addition, we also obtain the temperature dependence of mass at the same time, which shows the behavior of nucleon mass below the dissociation temperature.

This paper is organized as follows. In Sec. II, we show the reliability of GEM on studying the dissociation problem of quarkonium by comparing our results for dissociation temperature with others. In Sec. III, we construct the potential of nucleon at finite temperature and apply GEM to solve the corresponding Schrödinger equation. In Sec. IV, we show the results in 2-flavor QCD. Section V contains discussions and conclusions.

\section{THE RESULTS ON DISSOCIATION TEMPERATURE OF QUARKONIUM}

Before studying the dissociation of nucleon, we test the reliability of GEM on studying dissociation problem of quarkonium by comparing our results for dissociation temperatures, obtained by using GEM, with the results obtained by other methods. To compare with Satz's results, the potential of quarkonium at finite temperature we use is the same as that of Satz's work [5]. The results for dissociation temperatures of charmonium and bottomonium in Ref. [5] and our results are listed in Tables I and II respectively, which show our results are consistent with that in Ref. [5] (see Appendix for a detailed calculation on dissociation temperature of quarkonium). So GEM can give accurate results on the dissociation temperature of quarkonium. Giving accurate binding energy and wave function [8] makes the GEM very suitable for studying the dissociation problem of quark bound states. In the following, we will use this method to calculate the dissociation temperature of a nucleon.

TABLE I. Dissociation temperature $T_{d} / T_{c}$ of charmonium in Ref. [5] and our results.

\begin{tabular}{lccc}
\hline \hline Charmonium & $1 \mathrm{~S}$ & $1 \mathrm{P}$ & $2 \mathrm{~S}$ \\
\hline Ref. [5] & 2.10 & 1.16 & 1.12 \\
Our Results & 2.06 & 1.16 & 1.13 \\
\hline \hline
\end{tabular}

TABLE II. Dissociation temperature $T_{d} / T_{c}$ of bottomonium in Ref. [5] and our results.

\begin{tabular}{lccccc}
\hline \hline Bottomonium & $1 \mathrm{~S}$ & $1 \mathrm{P}$ & $2 \mathrm{~S}$ & $2 \mathrm{P}$ & $3 \mathrm{~S}$ \\
\hline Ref. [5] & $>4.0$ & 1.76 & 1.60 & 1.19 & 1.17 \\
Our Results & 5.81 & 1.71 & 1.56 & 1.18 & 1.17 \\
\hline \hline
\end{tabular}

\section{FORMALISM}

\section{A. Constituent quark model}

The constituent quark model, where multigluon degrees of freedom are eliminated in favor of confined constituent quarks with effective masses coming from dynamical chiral symmetry breaking, is the simplest and most successful QCD-inspired quark model. It has achieved a success in describing both the hadron spectra and the hadron-hadron interactions. In a nonrelativistic quark model, baryons are formed by three constituent quarks, which are confined by a confining potential and interact with each other by residual two-body interactions [15]. The potential of baryon can be described by a sum of the potential of corresponding two-quark system. For the potential of twoquark system, the study of heavy meson spectra and lattice calculation results suggested a linear form of confining interaction for the short-range part. Apart from the confining interaction, the interaction obtained from the onegluon exchange was derived long ago by Rujula et al. [16]. Work in Refs. [16-19] shows these two parts can give a good description of the baryon ground states. So we just consider the two parts (neglecting the part given by the Goldstone-boson exchanges) in our potential model. The details of the model can be found in Refs. [20,21]. To calculate the dissociation temperature of the nucleon, we will extend this quark model from zero temperature to finite temperature by extending the interquark potential at zero temperature to that at finite temperature. Because we have no idea for considering the spin-part of the interquark potential at finite temperature, we will neglect this part in the present work.

Thus the potential of a $q \bar{q}$ system is written as

$$
V_{q \bar{q}}(r)=-\frac{\alpha}{r}+\sigma r+\lambda,
$$

where $\alpha$ is the coupling constant, and $\sigma$ is the string tension. For Cornell potential, i.e., $V=-\frac{\alpha}{r}+\sigma r$, in lattice QCD, Kaczmarek's work [13] shows that the potential of diquark system is about half of that of corresponding quarkantiquark system, i.e., $V_{q q}=\frac{1}{2} V_{q \bar{q}}$ (obeying Casimir scaling). Thus our Hamiltonian is written as

$$
\begin{gathered}
H=\sum_{i=1}^{3}\left(m_{i}+\frac{\boldsymbol{p}_{i}^{2}}{2 m_{i}}\right)-T_{c m}+\sum_{1=i<j}^{3} \frac{1}{2} V\left(r_{i j}\right)+V_{\text {con }}, \\
V\left(r_{i j}\right)=-\frac{\alpha}{r_{i j}}+\sigma r_{i j},
\end{gathered}
$$

where $m_{i}$ is the constituent quark mass of the $i$ th quark, $V_{\text {con }}$ is a constant parameter and $T_{c m}$ is the c.m. kinetic energy. $\boldsymbol{r}_{i j}=\boldsymbol{r}_{i}-\boldsymbol{r}_{j}$ is the relative motion coordinate. In this model, the mass of light quark (u and d quark) we use is $300 \mathrm{MeV}$. For the potential, we choose two sets of 
TABLE III. Parameters in the potential model.

\begin{tabular}{lccc}
\hline \hline & $\alpha$ & $\sqrt{\sigma}[\mathrm{GeV}]$ & $V_{\text {con }}[\mathrm{GeV}]$ \\
\hline Set I & $\frac{\pi}{12}$ & 0.219 & -0.649 \\
Set II & $\frac{\pi}{11}$ & 0.217 & -0.633 \\
\hline \hline
\end{tabular}

parameters listed in Table III in order to get some insight about the sensitivity associated with the potential model.

Solving the corresponding Schrödinger equation,

$$
H \Psi_{\text {total }}=E \Psi_{\text {total }},
$$

with GEM, we get the mass $E$ and the corresponding wave function $\Psi_{\text {total }}$. We define the radius of nucleon as

$$
R=\frac{1}{3} \sum_{i=1}^{3} \sqrt{\left\langle r_{i}^{2}\right\rangle}
$$

with

$$
\left\langle r_{i}^{2}\right\rangle=\int \Psi_{\mathrm{total}}^{*} r_{i}^{2} \Psi_{\text {total }} d \tau
$$

where $r_{i}$ is the distance from the center of nucleon to the $i$-th quark. Then we calculate the radius of nucleon by using the obtained wave function. The resulting mass and radius of nucleon are about $939 \mathrm{MeV}$ and $0.840 \mathrm{fm}$ in this two cases, respectively, while the corresponding experimental data are about $939 \mathrm{MeV}$ and $0.841 \mathrm{fm}$. We see this model gives a good estimate for the properties of nucleon although the spin-dependent part of the potential is neglected. So it is reasonable for us to use this potential model to study the dissociation of the nucleon. Of course, we need to notice that the spin-dependent part plays an important role in the baryon spectrum.

The above calculation is performed at zero temperature. At high temperature, the interaction between quarks is screened. To study the dissociation of the nucleon, the quark model must be extended from zero temperature to finite temperature.

\section{B. Wave function}

In our work, GEM is used to solve the Schrödinger equation. In expressing three-quark wave functions, three sets of Jacobi coordinates (Fig. 1), called channels, are introduced. The Jacobi coordinates in each channel $c(c=1,2,3)$ are defined as

$$
\begin{gathered}
\boldsymbol{r}_{c}=\boldsymbol{x}_{j}-\boldsymbol{x}_{k}, \\
\boldsymbol{R}_{c}=\boldsymbol{x}_{i}-\frac{m_{j} \boldsymbol{x}_{j}+m_{k} \boldsymbol{x}_{k}}{m_{j}+m_{k}},
\end{gathered}
$$
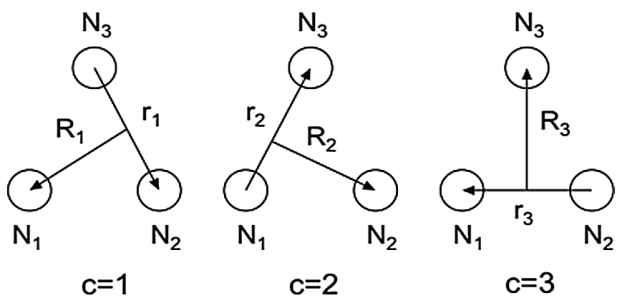

FIG. 1. Three sets of Jacobi coordinates for three-body system [8].

where $\boldsymbol{x}_{i}$ is the coordinate of the $i$ th quark and $(i, j, k)$ are given by Table IV.

The total wave function is described as a sum of amplitudes of three rearrangement channels $(c=1-3)$

$$
\Psi_{\text {total }}^{J M}=\sum_{c, \alpha} C_{c, \alpha} \Psi_{J M, \alpha}^{(c)}\left(\boldsymbol{r}_{c}, \boldsymbol{R}_{c}\right),
$$

where the index $\alpha$ represents $(s, S, l, L, I, n, N)$. Here $s$ is the spin of the $(i, j)$ quark pair, $S$ is the total spin, $l$ and $L$ are the orbital angular momentum for the coordinate $\boldsymbol{r}$ and $\boldsymbol{R}$, respectively, and $I$ is the total orbital angular momentum. The wave function for channel $c$ is given by

$$
\Psi_{J M, \alpha}^{(c)}\left(\boldsymbol{r}_{c}, \boldsymbol{R}_{c}\right)=\phi_{c} \otimes\left[X_{S, s}^{(c)} \otimes \Phi_{l, L, I}^{(c)}\right]_{J M} \otimes H_{T, t}^{(c)},
$$

where $\phi_{c}, X_{S, s}, \Phi_{l, L, I}$, and $H_{T, t}$ are the color wave function, the spin wave function, the orbital wave function, and the isospin wave function. One can refer [22] for derails. The orbital wave function $\Phi_{l, L, I}^{(c)}$ is expanded in terms of the Gaussian basis functions written in Jacobi coordinates $\boldsymbol{r}_{c}$ and $\boldsymbol{R}_{c}$ :

$$
\begin{gathered}
\Phi_{l, L, I}^{(c)}=\left[\phi_{l}^{(c)}\left(\boldsymbol{r}_{c}\right) \psi_{L}^{(c)}\left(\boldsymbol{R}_{c}\right)\right]_{I}, \\
\phi_{l m}^{(c)}\left(\boldsymbol{r}_{c}\right)=N_{n l} r_{c}^{l} e^{-\nu_{n} r_{c}^{2}} Y_{l m}\left(\hat{\boldsymbol{r}}_{c}\right), \\
\psi_{L M}^{(c)}\left(\boldsymbol{R}_{c}\right)=N_{N L} R_{c}^{L} e^{-\lambda_{N} R_{c}^{2}} Y_{L M}\left(\hat{\boldsymbol{R}}_{c}\right),
\end{gathered}
$$

with the range parameters, $\nu_{n}$ and $\lambda_{N}$, chosen as

$$
\begin{gathered}
\nu_{n}=1 / r_{n}^{2}, \quad r_{n}=r_{1} a^{n-1}\left(n=1, \ldots, n_{\max }\right), \\
\lambda_{N}=1 / R_{N}^{2}, \quad R_{N}=R_{1} A^{N-1}\left(N=1, \ldots, N_{\max }\right) .
\end{gathered}
$$

TABLE IV. The quark assignments $(i, j, k)$ for the Jacobi channels.

\begin{tabular}{llll}
\hline \hline Channel & $\mathrm{i}$ & $\mathrm{j}$ & $\mathrm{k}$ \\
\hline 1 & 1 & 2 & 3 \\
2 & 2 & 3 & 1 \\
3 & 3 & 1 & 2 \\
\hline \hline
\end{tabular}


In Eqs. (12) and (13), $N_{n l}\left(N_{N L}\right)$ denotes the normalization constant of the Gaussian basis. The coefficients $C_{c, \alpha}$ of the variational wave function, Eq. (9), are determined by Rayleight-Ritz variational principle. In this calculation, we set the width of the range parameters and the number of basis functions large enough to ensure the reliability of the calculation.

\section{Potential model at finite temperature}

To calculate the dissociation temperature of nucleon, we need the interquark potential of nucleon in a hot medium. However, this potential is not yet well understood so far. Only for very small distances or very high temperatures one can calculate it perturbatively [3]. On the other hand, the free energy can be calculated in lattice QCD [10,11]. In a general case, the potential we need is different from the free energy and it is located between the following two limits [23]. One is the limit of rapid dissociation compared to the timescale of the heat exchange with the medium. In this case the nucleon has no time to exchange heat with the hot medium, and the interquark potential is just the internal energy, i.e., $V=F+s T$ with $s$ being the entropy density $s=-\partial F / \partial T$. The other is the limit of slow dissociation, the nucleon has enough time to exchange heat with the hot medium, and the potential is the free energy $V=F$. Noting the difference between internal energy and free energy lies in the contribution of entropy, the two limits are easy to understand. Because of having not known the realistic potential, one generally only consider the two limits. One can describe the collisional processes by a fireball model [24-26]. After the collision, the temperature of fireball is very high at a very short time in the beginning and deconfinement of hadron occurs at the same time. Then the temperature of fireball decreases until the phase transition temperature and the matter in fireball undergoes the phase transition from the deconfinement phase to the hadron phase, called hadronization. So we believe that the process of deconfinement can be considered as an adiabatic process. According to this argument, the choice using the internal energy as the potential is more reasonable. The studies about the dissociation of quarkonium support this argument [5,27]. For example, in Ref. [27], the authors calculate the in-medium charmonium properties in the two limits and the results show using the internal energy as the interquark potential tends to better reproduce current date on transverse momentum spectra at both SPS (the Super Proton Synchrotron) and RHIC (Relativistic Heavy Ion Collider). So in our work, we will use the internal energy as the interquark potential.

The interquark potential of nucleon at zero temperature has been discussed above and its parameters have been determined by fitting the properties of nucleon. To determine the dissociation temperature of nucleon, we need its potential at finite temperature, i.e., $V_{q q q}(\boldsymbol{r}, T)$ (the index q represents $\mathrm{u}$ or $\mathrm{d}$ quark). There is not screening effect for the constant term of potential, i.e., $V_{\text {con }}$ in Eq. (2). So we only need to consider the screening effect on the Cornell potential term, i.e., the third term of the right side in Eq. (2). As mentioned above, we assume that the interquark potential is just the internal energy

$$
\begin{aligned}
V_{q q q}(\boldsymbol{r}, T) & =U_{q q q}(\boldsymbol{r}, T) \\
& =F_{q q q}(\boldsymbol{r}, T)+s T,
\end{aligned}
$$

where $s$ is the entropy density $s=-\partial F_{q q q} / \partial T$. In Refs. [10,14,28], Kaczmarek's works show that the singlet free energy of the heavy three-quark systems $\left(F_{q q q}^{1}\right)$ are well described by the sum of the antitriplet free energy of corresponding three diquark systems $\left(F_{q q}^{\overline{3}}\right)$ plus self-energy contributions of the three quarks at high temperature in 2-flavor QCD. It is expressed as

$$
F_{q q q}^{1}(\boldsymbol{R}, T) \simeq \sum_{i<j} F_{q q}^{\overline{3}}\left(R_{i j}, T\right)-3 F_{q}(T),
$$

where the self-energy $F_{q}(T)=\frac{1}{2} F_{q q}^{\overline{3}}(\infty, T)$ and $\boldsymbol{R}$ represents $\left(\boldsymbol{R}_{12}, \boldsymbol{R}_{13}, \boldsymbol{R}_{23}\right)$. In Ref. [13], Kaczmarek's work suggests a simple relation between the free energy of the antitriplet $q q$ state and that of the singlet $q \bar{q}$ state

$$
F_{q \bar{q}}^{1}(r, T) \simeq 2\left(F_{q q}^{\overline{3}}(r, T)-F_{q}(T)\right) .
$$

The analytical form of $F_{q \bar{q}}^{1}(r, T)$ can be obtained based on studies of screening in Debye-Hückel theory. It is written as [3]

$$
\begin{aligned}
F_{q \bar{q}}^{1}(r, T)= & -\frac{\alpha}{r}\left[e^{-\mu r}+\mu r\right]+\frac{\sigma}{\mu}\left[\frac{\Gamma(1 / 4)}{2^{3 / 2} \Gamma(3 / 4)}\right. \\
& \left.-\frac{\sqrt{\mu r}}{2^{3 / 4} \Gamma(3 / 4)} K_{1 / 4}\left[(\mu r)^{2}+\kappa(\mu r)^{4}\right]\right],
\end{aligned}
$$

where the screening mass $\mu$ and the parameter $\kappa$ are temperature-dependent, and $K_{1 / 4}[x]$ is the modified Bessel function. We can determine the T-dependent $\mu$ and $\kappa$ by fitting $F_{q \bar{q}}^{1}(r, T)$ to the lattice results obtained in 2-flavor QCD [11]. At $r=\infty$, the free energy $F_{q \bar{q}}^{1}(T)$ is written as

$$
F_{q \bar{q}}^{1}(T)=\frac{\sigma}{\mu(T)} \frac{\Gamma(1 / 4)}{2^{3 / 2} \Gamma(3 / 4)}-\alpha \mu(T) .
$$

Thus, the form of $\mu(T)$ is given as function of $F_{q \bar{q}}^{1}(T)$

$$
\mu(T)=\frac{\left[\sqrt{F_{q \bar{q}}^{1}(T)^{2}+4 \sigma \alpha \frac{\Gamma(1 / 4)}{2^{3 / 2} \Gamma(3 / 4)}}-F_{q \bar{q}}^{1}(T)\right]}{2 \alpha} .
$$

To determine the temperature dependence of $\mu$, we fit this $F(T)$ to the lattice data for $r=\infty$. Once we have the 


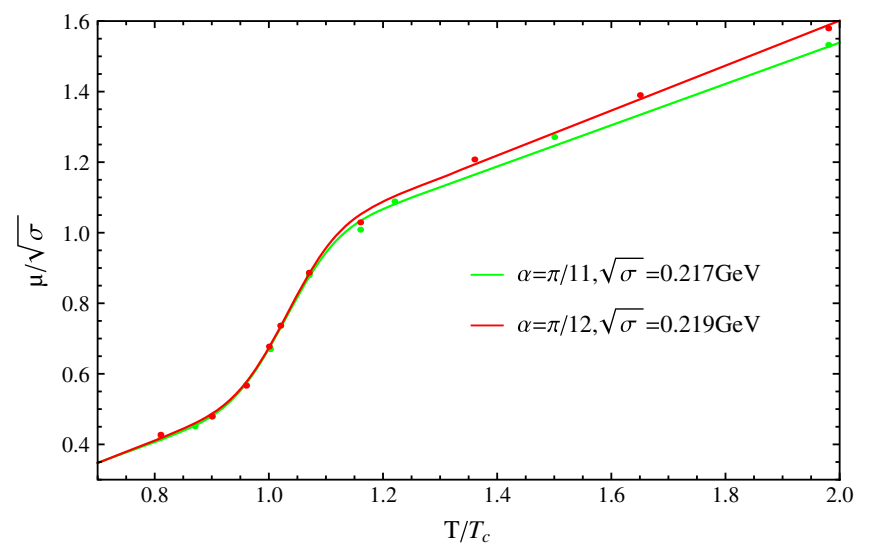

FIG. 2. Results for $\mu(T)$ in 2-flavor QCD.

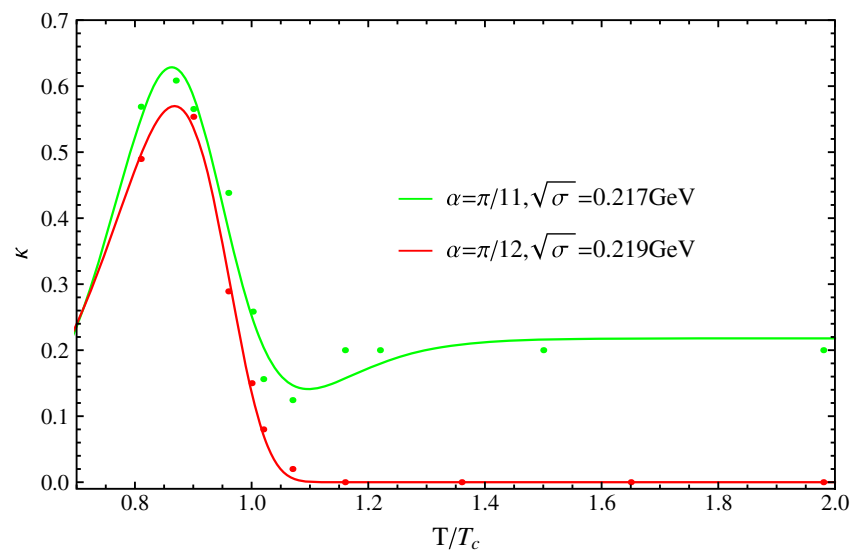

FIG. 3. Results for $\kappa(T)$ in 2-flavor QCD.

temperature dependence of $\mu(T)$, we fit Eq. (19) to the lattice data to obtain $\kappa(T)$. It should be pointed out that Kaczmarek's work gets $T_{c}=202 \mathrm{MeV}$ in 2-flavor QCD [11]. The fitted results for the temperature dependence of $\mu(T)$ and $\kappa(T)$ are shown in Figs. 2 and 3, respectively. In Fig. 4, we also show our fit curves (solid lines) together with the lattice results. We can see that our form of the free energy $F_{q \bar{q}}^{1}(r, T)$ fits the lattice data quite well for all $r$ and in a broad range of temperatures from $0.8 T_{c}$ to $2 T_{c}$ in the two cases.

Combining Eq. (17) with Eq. (18), we get a relation between the singlet free energy of the heavy three-quark system and the singlet free energy of $q \bar{q}$

$$
F_{q q q}^{1}(\boldsymbol{R}, T) \simeq \sum_{i<j} \frac{1}{2} F_{q \bar{q}}^{1}\left(R_{i j}, T\right) .
$$

In Kaczmarek's works, these conclusions are obtained in the case of the heavy quark system. In our work, we extend these conclusions to the light quark system. To obtain the binding energy of nucleon, we define an effective potential as

$$
\tilde{V}_{q q q}(\boldsymbol{R}, T)=V_{q q q}(\boldsymbol{R}, T)-V_{q q q}(\infty, T) .
$$
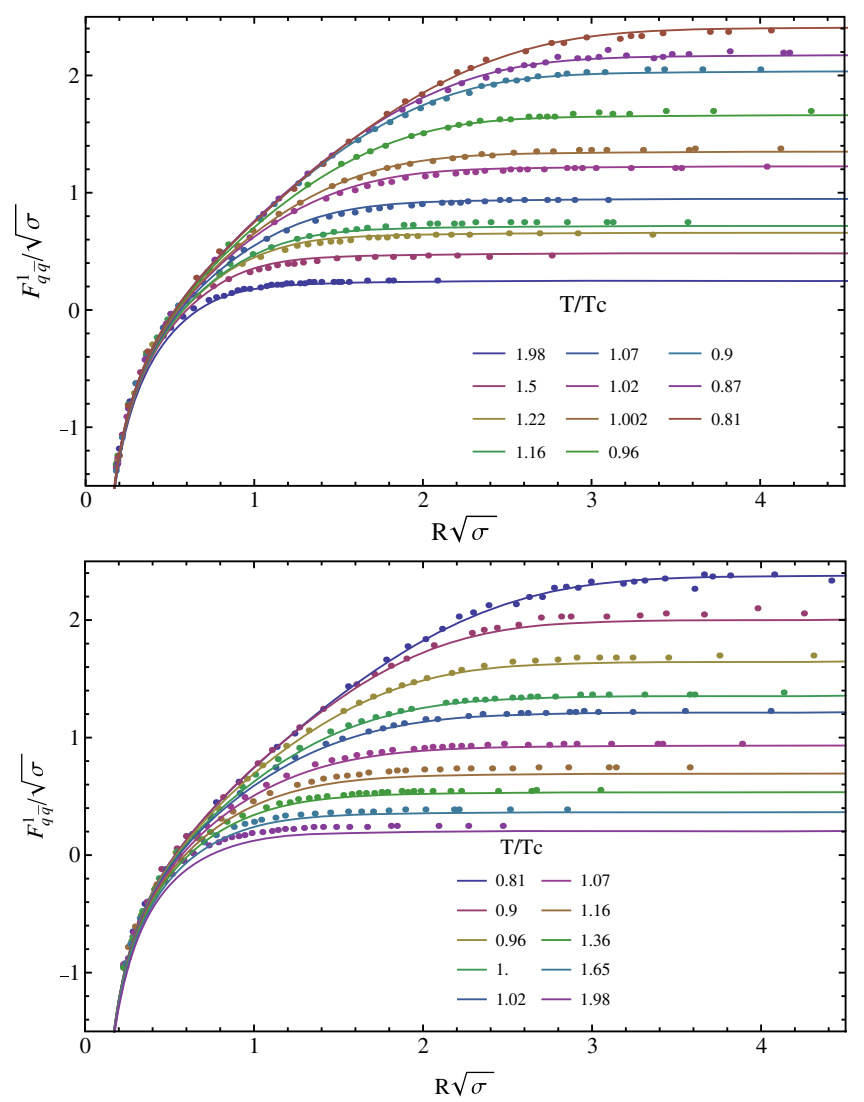

FIG. 4. Fits to the lattice results for free energy $F_{q \bar{q}}^{1}$ in 2-flavor QCD: the upper figure shows the result for the case: $\alpha=\frac{\pi}{11}$ (data from [11]), and the lower figure shows the result for another case: $\alpha=\frac{\pi}{12}$ (data from [5]).

Combining Eqs. (16), (22), (23), we get a relation between the effective potential and the singlet free energies of $q \bar{q}$

$\tilde{V}_{q q q}(\boldsymbol{R}, T)=\sum_{i<j} \frac{1}{2}\left(\tilde{F}_{q \bar{q}}^{1}\left(R_{i j}, T\right)-T \frac{\partial \tilde{F}_{q \bar{q}}^{1}\left(R_{i j}, T\right)}{\partial T}\right)$

with

$$
\tilde{F}_{q \bar{q}}^{1}\left(R_{i j}, T\right)=F_{q \bar{q}}^{1}\left(R_{i j}, T\right)-F_{q \bar{q}}^{1}(\infty, T) .
$$

Replacing the potential term, $\sum_{1=i<j}^{3} \frac{1}{2} V\left(r_{i j}\right)+V_{\text {con }}$, in Eq. (2) with this effective potential $\tilde{V}_{q q q}(\boldsymbol{R}, T)$ and deleting the mass term, we get a new Hamiltonian for nucleon at finite temperature written as

$$
H_{\text {new }}=\sum_{i=1}^{3} \frac{\boldsymbol{p}_{i}^{2}}{2 m_{i}}-T_{c m}+\tilde{V}_{q q q}(\boldsymbol{R}, T) .
$$

The relevant Schrödinger equation now becomes

$$
H_{\text {new }} \Psi_{\text {total }}^{J M}=\epsilon(T) \Psi_{\text {total }}^{J M} \text {, }
$$


where

$$
\epsilon(T)=M-3 m_{q}-V_{q q q}(\infty, T) .
$$

Solving this Schrödinger equation, we obtain the binding energy $\Delta E(T)(=-\epsilon(T))$ and the corresponding wave function at temperature T. Once we have the wave function, we can calculate the T-dependent radius according to Eq. (5). Replacing the potential term, $\sum_{1=i<j}^{3} \frac{1}{2} V\left(r_{i j}\right)$, in Eq. (2) with the potential $V_{q q q}(\boldsymbol{R}, T)$ in Eq. (16), we obtain the temperature dependence of nucleon mass in the same way.

\section{NUMERICAL RESULTS}

In Fig. 5, we show the binding energies behavior for the nucleon in the two cases mentioned above. We can see the two lines have similar behavior. When the binding energy vanishes, the nucleon no longer exists, so that $\Delta E(T)=0$ determines the dissociation temperature. From Fig. 5, we get the dissociation temperatures in the two cases are about $1.16 T_{c}$ and $1.17 T_{c}$, respectively. In Fig. 6, we show the corresponding nucleon radii. The nucleon radius approach infinity near the dissociation temperature. The dissociation temperature determined from Fig. 6 is consistent with that determined from Fig. 5. It is seen that the divergence of the radii defines quite well the different dissociation points in the two cases. There are slight differences between the results of the two cases. The resulting dissociation temperature just changes one percent while the parameter $\alpha$ changes about ten percent, which means that the result is insensitive to the parameters of our potential model. If there is no significant change on parameter $\alpha$, the resulting dissociation temperature has no significant change. Figure 7 shows the resulting mass behavior for the nucleon. With the temperature increasing from $0.9 T_{c}$ to the dissociation temperature, the nucleon mass increases before $T_{c}$ and then decreases. The result is in agreement with that of Refs. [29,30]. From Figs. 5 and 6, we can see that when

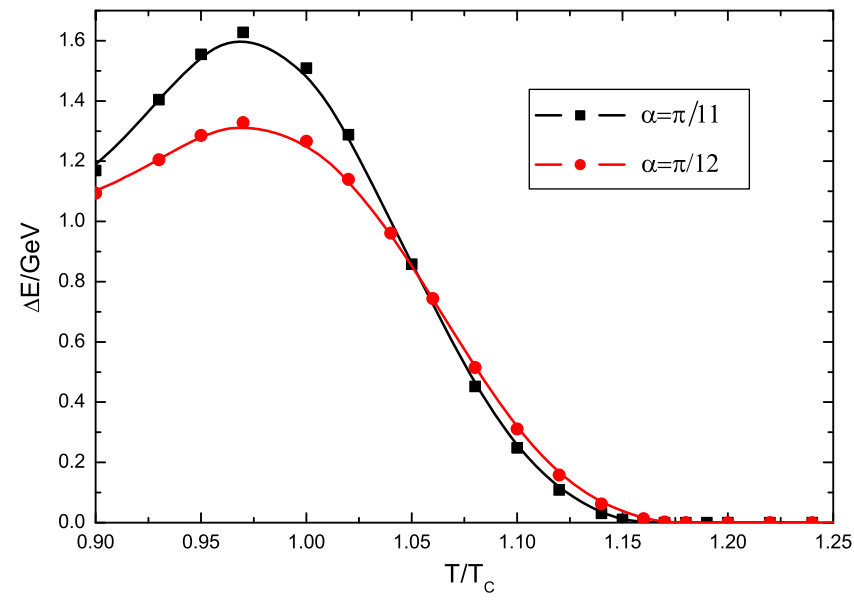

FIG. 5. T-dependence of binding energy in 2-flavor QCD.

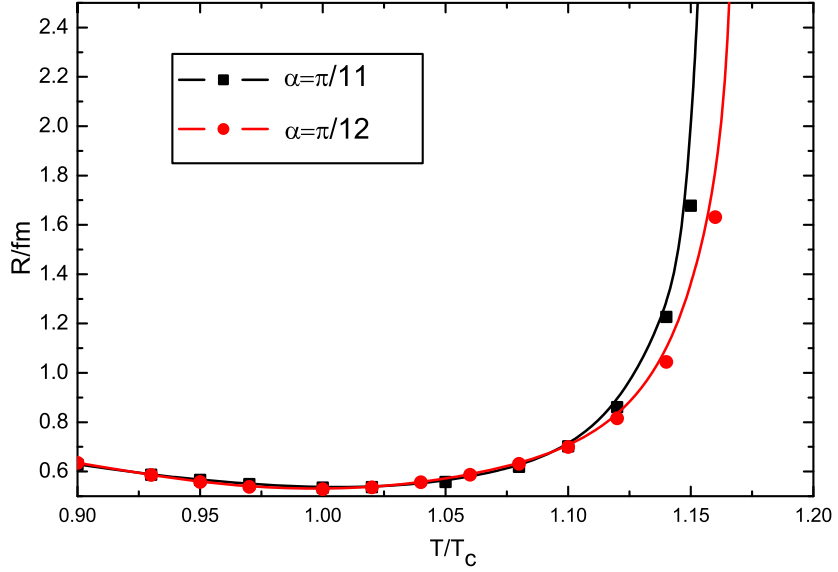

FIG. 6. T-dependence of radii in 2-flavor QCD.

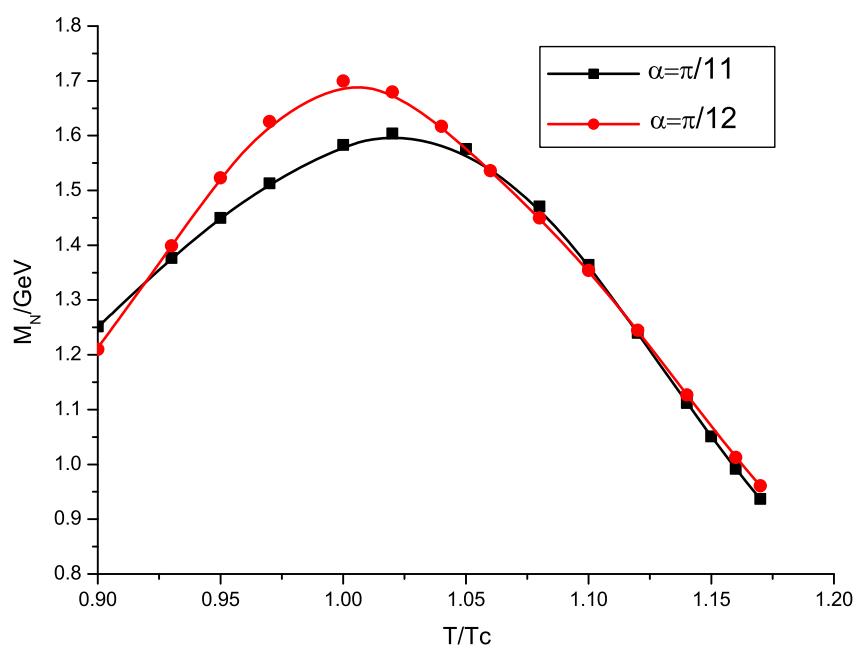

FIG. 7. T-dependence of mass in 2-flavor QCD.

temperature increases from $0.9 T_{c}$ to $T_{c}$, the binding energy increases and the radii decreases, which means the nucleon becomes more stable, and after $T_{c}$, the behavior of radii and binding energies means the nucleon tends to melt.

\section{DISCUSSION AND CONCLUSIONS}

The free energy of quark-antiquark system we construct based on Debye-Hückel theory fits the lattice data quite well for all $r$ from $0.8 T_{c}$ to $2 T_{c}$. According to Kaczmarek's works, we can get a relation between the singlet free energy of heavy $q q q$ system $F_{q q q}^{1}$ and the singlet free energy of heavy $q \bar{q}$ system $F_{q \bar{q}}^{1}$, written as $F_{q q q}^{1}(\boldsymbol{R}, T) \simeq$ $\sum_{i<j} \frac{1}{2} F_{q \bar{q}}^{1}\left(R_{i j}, T\right)$. In our model, the nucleon mass increases as temperature increases from $0.9 T_{c}$ to $T_{c}$, but after the point $T_{c}$ it decreases. The behavior of binding energy is similar to the mass. On the contrary, the corresponding radius decreases as the temperature increases from $0.9 T_{c}$ to $T_{c}$, which means the nucleon is becoming more stable, and 
after the point $T_{c}$ the radius increases up to infinity near the dissociation temperature, which means the nucleon tend to melt. The dissociation temperatures of nucleon in 2-flavor QCD in our calculation are about $1.16 T_{c}$ for $\alpha=\frac{\pi}{11}$ and $1.17 T_{c}$ for $\alpha=\frac{\pi}{12}$. The result is insensitive to the model's parameters. Our result show that the deconfinement of nucleon occurs after $T_{c}$.

It should be pointed out that in our present work, we neglect the spin-dependent part in our potential model, which may have some effect on the resulting dissociation temperature of nucleon. The reason why we ignore the contribution of the spin-dependent part in this paper is that it is difficult for us to consider the screening effect on the potential of nucleon in a hot medium when we take the spin-dependent part into account. In addition, the collisional processes can be described by a Fireball model, where the nucleon is not simply captured by a real potential we used in our work. For the fireball, its volume is finite and the baryon density is not vanishing in fact. However, the case we consider is only that the volume is infinite and the baryon density is vanishing. So there are some effects, arising from finite volume, magnetic field, finite baryon density and so on, contributing to baryon dissociation. It is undoubted that such work deserves our progressive consideration.

\section{ACKNOWLEDGMENTS}

This work is supported in part by the National Natural Science Foundation of China (under Grants No. 11475085, No. 11535005 , No. 11690030 , No. 11775118) and by Nation Major State Basic Research and Development of China (2016YFE0129300).

\section{APPENDIX: THE CALCULATION ON DISSOCIATION TEMPERATURE OF QUARKONIUM}

We have given our results for dissociation temperature of quarkonium in Sec. II. Here we present the calculation in detail. For the quarkonium, the potential we use is the Cornell potential. The Hamiltonian for quarkonium is written as

$$
H=\frac{\boldsymbol{p}^{2}}{2 \mu_{12}}+V_{q \bar{q}}(\boldsymbol{r}),
$$

where $\boldsymbol{p}=\boldsymbol{p}_{2}-\boldsymbol{p}_{1}$ and $\mu_{12}$ is the reduced mass. The parameters taken from Ref. [5] are: $m_{c}=1.25 \mathrm{GeV}$, $\sqrt{\sigma}=0.445 \mathrm{GeV}, \alpha=\pi / 12$, and $m_{b}=4.65 \mathrm{GeV}$. Then we can construct the corresponding free energy at finite temperature based on Debye-Hückel theory as having done above. For simplicity, we neglect the term $\kappa(\mu r)^{4}$ in Eq. (19). This is reasonable because we can see $\kappa$ is close to zero from $0.8 T_{c}$ to $2 T_{c}$. We can obtain the T-dependent parameter $\mu(T)$ by fitting Eq. (19) to lattice data. According to the potential model mentioned above, we can obtain the potential of quarkonium at finite temperature. Then, the relevant Schrödinger equation is written as

$$
\begin{aligned}
& \left(-\frac{1}{2 \mu_{12}} \nabla^{2}+V_{q \bar{q}}(r, T)-V_{q \bar{q}}(\infty, T)\right) \psi_{i}(r, T) \\
& \quad=\epsilon_{i}(T) \psi_{i}(r, T),
\end{aligned}
$$

where the index $i$ represents a quarkonium state and $\Delta E_{i}(T)\left(=-\epsilon_{i}(T)\right)$ is the responding binding energy at temperature T. According to Ref. [8], we expand the total wave function in terms of a set of basis functions as

$$
\psi_{l m}=\sum_{n=1}^{n_{\max }} c_{n} \phi_{n l m},
$$

with

$$
\begin{gathered}
\phi_{n l m}(r)=\phi_{n l}(r) Y_{l m}(\hat{\boldsymbol{r}}), \\
\phi_{n l}(r)=N_{n l} r^{l} e^{-v_{n} r^{2}},
\end{gathered}
$$

where $N_{n l}$ is the normalization constant. The RayleightRitz variational principle leads to a generalized matrix eigenvalue problem

$$
\sum_{n^{\prime}=1}^{n_{\max }}\left(H_{n n^{\prime}}-E N_{n n^{\prime}}\right) c_{n^{\prime} l}=0 .
$$

Therefore, we can obtain the eigenvalues and the corresponding wave functions of both ground state and excited states. We define the root mean square (RMS) radii of quarkonium as:

$$
\sqrt{\left\langle r_{i}\right\rangle}=\left[\int \psi_{i}^{*} r^{2} \psi_{i} d \tau\right]^{1 / 2} .
$$

Then we can use the resulting wave function to calculate the temperature-dependent radii. 
[1] J. M. Torres-Rincon, B. Sintes, and J. Aichelin, Phys. Rev. C 91, 065206 (2015).

[2] T. Matsui and H. Satz, Phys. Lett. B 178, 416 (1986).

[3] S. Digal, O. Kaczmarek, F. Karsch, and H. Satz, Eur. Phys. J. C 43, 71 (2005).

[4] F. Karsch, M. Mehr, and H. Satz, Z. Phys. C 37, 617 (1988).

[5] H. Satz, J. Phys. G 32, R25 (2006).

[6] W. M. Alberico, A. Beraudo, A. De Pace, and A. Molinari, Phys. Rev. D 72, 114011 (2005).

[7] Q. Zhen, L. Yun-Peng, and Z. Peng-Fei, Chin. Phys. Lett. 29, 031201 (2012).

[8] E. Hiyama, Y. Kino, and M. Kamimura, Prog. Part. Nucl. Phys. 51, 223 (2003).

[9] M. Qi, W. Qian, C. Peng, P. Jialun, and Z. Hongshi, Chin. Phys. C 42, 083103 (2018).

[10] O. Kaczmarek, F. Karsch, P. Petreczky, and F. Zantow, Phys. Lett. B 543, 41 (2002).

[11] O. Kaczmarek and F. Zantow, Phys. Rev. D 71, 114510 (2005).

[12] V. V. Dixit, Mod. Phys. Lett. A 05, 227 (1990).

[13] M. Döring, K. Hübner, O. Kaczmarek, and F. Karsch, Phys. Rev. D 75, 054504 (2007).

[14] K. Hübner, F. Karsch, O. Kaczmarek, and O. Vogt, Phys. Rev. D 77, 074504 (2008).

[15] T. Yoshida, E. Hiyama, A. Hosaka, M. Oka, and K. Sadato, Phys. Rev. D 92, 114029 (2015).
[16] A. De Rújula, H. Georgi, and S. L. Glashow, Phys. Rev. D 12, 147 (1975).

[17] N. Isgur and G. Karl, Phys. Rev. D 18, 4187 (1978).

[18] N. Isgur and G. Karl, Phys. Rev. D 19, 2653 (1979).

[19] N. Isgur and G. Karl, Phys. Rev. D 20, 1191 (1979).

[20] L. Y. Glozman, Z. Papp, W. Plessas, K. Varga, and R. F. Wagenbrunn, Phys. Rev. C 57, 3406 (1998).

[21] R. K. Bhaduri, L. E. Cohler, and Y. Nogami, Phys. Rev. Lett. 44, 1369 (1980).

[22] G. Yang, J. Ping, and J. Segovia, Few-Body Syst. 59, 113 (2018).

[23] E. V. Shuryak and I. Zahed, Phys. Rev. D 70, 054507 (2004).

[24] G. D. Westfall, J. Gosset, P. J. Johansen, A. M. Poskanzer, W. G. Meyer, H. H. Gutbrod, A. Sandoval, and R. Stock, Phys. Rev. Lett. 37, 1202 (1976).

[25] K. A. Olive, Phys. Lett. B 89, 299 (1980).

[26] Y. Burnier, O. Kaczmarek, and A. Rothkopf, J. High Energy Phys. 12 (2015) 101.

[27] X. Zhao and R. Rapp, Phys. Rev. C 82, 064905 (2010).

[28] M. Döring, K. Hübner, O. Kaczmarek, and F. Karsch, Phys. Rev. D 75, 054504 (2007).

[29] M. Abu-Shady, Int. J. Theor. Phys. 50, 1372 (2011).

[30] V. Bernard and U.-G. Meissner, Phys. Rev. C 39, 2054 (1989). 